\title{
DP: dialisi marginale?
}

\author{
Michele Giannattasio, Roberto La Rosa
}

\author{
Unità Operativa di Nefrologia e Dialisi, Ospedale Santa Maria degli Angeli \\ Azienda USL Bari 5, Putignano (Bari)
}

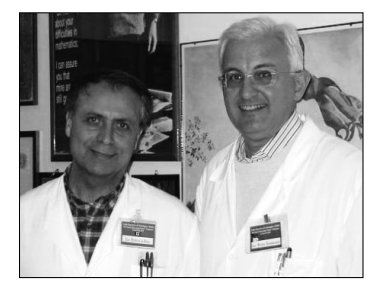

Roberto La Rosa e Michele Giannattasio

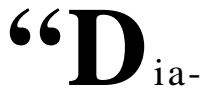

lisi peritoneale: Principessa o Cenerentola?" L'originale interrogativo posto alcuni anni orsono sulla validità della dialisi peritoneale (DP) nell' ambito dei trattamenti sostitutivi della funzione renale ancora oggi, purtroppo, è oggetto di discussione, nonostante $\mathrm{i}$ progressi e $\mathrm{i}$ risultati ottenuti negli ultimi 15-20 anni dalla DP siano indiscutibili. Ciononostante la DP, anche se con percentuali di penetrazione molto differenti nei vari Paesi (Nuova Zelanda $56 \%$, Canada 37\%, Australia 31\%, USA 14\%, Europa 12\%, Giappone $5.6 \%$ ) continua ancora oggi a svolgere un ruolo da "Cenerentola", nomignolo molto espressivo e non casuale.

È noto che la scelta della terapia dialitica è complessa perché deve tenere conto degli aspetti clinici, di quelli organizzativi ma anche e in maniera sempre più pressante di quelli economici; inoltre, nel successo di una particolare tecnica di dialisi sicuramente hanno importanza l'esperienza, l'entusiasmo e l'impegno profuso in quella specifica tecnica dallo staff medico e infermieristico.

La validità della DP in Italia e nel mondo è ben nota ed è molto limitata la percentuale di nefrologi che ne ignora le potenzialità. Per la buona riuscita di un programma di DP sono sicuramente necessarie conoscenze specifiche e personale medico e infermieristico incline alla metodica oltre che ben addestrato, motivato e possibilmente dedicato esclusivamente alla DP. È di fondamentale importanza anche il coinvolgimento culturale di tutto il gruppo dell'Unità Operativa (U.O.) di Nefrologia e Dialisi ed anche di quelle persone (medici, infermieri, tecnici ecc.) che non svolgono la propria attività nell'ambulatorio di DP; tutto il "gruppo" deve essere aggiornato sui più recenti orientamenti di scelta del trattamento dialitico che sono quelli di considerare la DP parte integrante del trattamento sostitutivo dell'uremia cronica (1). Questo coinvolgimento del "gruppo" e, quindi, la sua crescita culturale impediranno di trasmettere, seppur inconsciamente, ai pazienti da avviare alla dialisi atteggiamenti denigratori o informazioni errate sulla metodica peritoneodialitica, legati alla scarsa conoscenza dei numerosi potenziali vantaggi che fanno della DP la tecnica di prima scelta del trattamento dell'uremia (2).

Tuttavia ancora oggi alcuni Centri nefrologici risentono del negativo influsso che ebbero agli inizi degli anni ' 80 gli scadenti risultati sulla sopravvivenza dei pazienti in CAPD: a quei tempi, infatti, la DP fu quasi completamente abbandonata e considerata una tecnica depurativa di livello inferiore all'ED. In tale contesto la DP non è proposta ovvero, a seguito d'esplicita richiesta, sconsigliata. Ciò, malgrado i numerosi problemi di tecnica siano stati superati tanto che anche l'incidenza di peritonite, pur rimanendo ancora oggi la causa maggiore di passaggio all'ED, si è notevolmente ridotta passando dai 6.3 episodi/paziente/anno nel 1980 agli attuali 0.5 episodi/paziente/anno (3). A questi pregiudizi, legati a un'inadeguata informazione, si somma il maggior impegno necessario per l'allestimento e il mantenimento di un programma di DP di buon livello: perciò un organico insufficiente di nefrologi e/o di infermieri professionali potrebbe rendere problematica l'organizzazione di tale programma. Quindi la disparità nell'utilizzo della DP sul territorio nazionale potrebbe essere spiegato da un insufficiente nume- 
ro di nefrologi in alcune aree; tuttavia in Europa non v'è correlazione tra numero di nefrologi per milione di popolazione (pmp) e penetrazione della DP. In Italia, in particolare, vi sono 66 nefrologi pmp con una penetrazione della metodica del 10-12\% contro 5.2 nefrologi pmp del Regno Unito che ha una penetrazione della DP del $45 \%$ nel 1995 (4).

Molti nefrologi, pertanto, preferiscono la più nota e meno problematica ED che da sola, non riesce a far fronte alla crescente richiesta di terapia dialitica, legata al progressivo incremento della popolazione uremica.

Non bisogna dimenticare, inoltre, gli aspetti economici. Il costo per anno della DP, in particolare della CAPD, è del $30 \%$ inferiore a quello dell'ED ospedaliera, in quanto i costi fissi e quelli indotti sono più bassi e il rapporto infermiere/pazienti è più basso rispetto a quello dell'ED. Il programma di DP, non essendo limitato dai posti-tecnici come l'ED, consente di poter aumentare il numero di pazienti in trattamento disponendo di minori risorse ma consentendo maggiori investimenti capitali. V'è, tuttavia, da considerare anche il problema delle tariffe: il minor utilizzo della DP in Germania e negli USA è dovuto ai più scadenti rimborsi delle prestazioni di DP. Per quanto attiene la situazione italiana le tariffe dialitiche ministeriali sono quasi tutte ampiamente sottostimate rispetto ai costi reali. È noto che queste tariffe in una fase successiva sono state modificate, in genere nel senso di una riduzione, dagli Assessorati Regionali alla Sanità; pertanto errate politiche regionali, che penalizzino pesantemente le tariffe della DP, potrebbero orientare le scelte dei nefrologi verso tecniche dialitiche di costo maggiore ma meglio retribuite, come quelle emodialitiche. L'introduzione di opportuni incentivi nella programmazione sanitaria a livello nazionale e regionale potrebbe contribuire ad aumentare il numero di pazienti da avviare alla DP.
È evidente che esaurita la disponibilità di posti-rene negli Ospedali e considerato anche lo scarso ausilio apportato dal settore trapianti, non restano che i Centri emodialitici privati i quali, sono particolarmente diffusi nel Centro-Sud dell'Italia, proprio in quelle aree nelle quali è bassa la penetrazione della DP.

"Cenerentola", quindi, non crediamo che mai diventerà "Principessa" perché gli interessi economici che gravitano nel mondo della dialisi e l'inerzia di molti nefrologi, difficilmente saranno superati dal buon senso che dovrebbe condurre a un maggior utilizzo della DP, intesa come dialisi "first choice", complementare all'ED e inserita nel programma integrato di terapia depurativa, chiamato Terapia Dialitica Integrata (5), che ogni Centro dialitico dovrebbe essere in grado di offrire poiché meglio rispecchia le necessità cliniche e riabilitative dei singoli pazienti.

\section{michele.giannattasio@libero.it}

\section{BIBLIOGRAFIA}

1.

Giannattasio M, La Rosa R. L'addestramento alla dialisi peritoneale domiciliare. Princìpi di dialisi peritoneale. II Edizione a cura di Scanziani R. e Surian M. SpringerVerlag Italia, Milano (in press).

2. Giannattasio M. Emodialisi o Peritoneodialisi come terapia di prima scelta dell'uremia cronica? Giorn It Nefrol 2000; 2: 161-9.

3. Fried L, Piraino B. Peritonitis. In Textbook of Peritoneal Dialysis. 2nd Edition Ed by Gokal R, Khanna R, Krediet R, Nolph K. Kluger Academic Publishers 2000; 545-64.

4. Mignon F, Michel C, Viron B. Why so much disparity of PD in Europe? Nephrol Dial Transplant 1998; 13: 1114-7.

5. Van Biesen L, Vanholder R, Veys N, Dhondt A, Lameire N. An evaluation of an integrative care approach for end-stage renal disease patients J Am Soc Nephrol 2000; 11: 116-125. 